\title{
Stochastic Stabilization of Linear Systems Driven by Reflecting Brownian Motion
}

\author{
Yanyan $\mathrm{Xie}^{1} \&$ Zhengping Shi ${ }^{1}$ \\ ${ }^{1}$ College of Mathematics and Computer Science, Fuzhou University, Fuzhou, China \\ Correspondence: Yanyan Xie, College of Mathematics and Computer Science, Fuzhou University, Fuzhou, Fujian, \\ China. Tel: 189-5049-2436. E-mail: xieyanyan0537@126.com
}

Received: September 4, 2012 Accepted: September 26, 2012 Online Published: October 17, 2012

doi:10.5539/ijsp.v1n2p198 URL: http://dx.doi.org/10.5539/ijsp.v1n2p198

\begin{abstract}
This paper investigates the perturbation of an unstable linear differential equation by random noise that is a reflecting Brownian motion. A sufficient almost sure exponential stability condition for the perturbed system is established and the corresponding sample Lyapunov exponent is estimated.
\end{abstract}

Keywords: stabilization, reflecting Brownian motion, Lyapunov exponent, semimartingale Itô's formula, almost surely exponentially stable

\section{Introduction}

As known to all, an unstable deterministic dynamical system of ordinary differential equations can be stabilized by random noise. In recent years, there have been lots of scholars devoted to study the phenomenon by taking various forms of noise, and the achievements are outstanding. Hasminskii (1981) as one of the early pioneers in stabilization chose two white noise to stabilize an certain linear system. Arnold et al. (1983) proved that a zero mean stationary parameter noise can make an unstable linear system stable. Deng et al. (2001) solved the stochastic stabilization problem of nonlinear systems using noise with unknown covariance. By adding a multidimensional Brownian motion to the nonlinear system, Mao $(1994,1997)$ studied the stability of perturbed system systematically and set up a general method of stochastic stabilization. Applebaum and Siakalli (2010) extended this general theory to the stochastic stabilization of dynamical systems driven by a more general Lévy process. As a transformation of Brownian motion, the reflecting Brownian motion has limited moving range. However, up to now, there has been no research about the perturbation of unstable systems when they are driven by this noise. The purpose of this paper is to attempt to study this case.

This paper focuses on an unstable linear ordinary differential equation that is added random disturbance-a reflecting Brownian motion and investigates the exponential stability of the perturbed system. Section 2 introduces the necessary preliminaries. Section 3 establishes the conditions to judge whether the perturbed system is stable. Finally, an example is given to show the fact that the reflecting Brown motion noise can stabilize an unstable linear system successfully.

\section{Preliminaries}

Let $(\Omega, \mathscr{F}, P)$ be a complete probability space with a right continuous and increasing filtration $\left\{\mathscr{F}_{t}\right\}_{t \geq 0}$ containing all $P$-null sets. Suppose that $B=(B(t), t \geq 0)$ is a $\mathscr{F}_{t}$ adapted standard Brownian motion taking values in $R$ on the probability space. If $Y(t)=|B(t)|$, then $Y=(Y(t), t \geq 0)$ is a $\mathscr{F}_{t}$ adapted reflecting Brownian motion on the half line $[0, \infty)$.

Consider the following one-dimensional linear systems

$$
\frac{d x(t)}{d t}=a x(t), \quad t \geq 0,
$$

where the constant $a>0$ and the initial value $x(0)=x_{0} \in R$ is given. Obviously the linear system (1) is unstable when $x_{0} \neq 0$. Now, to make this linear system stable, perturb the linear system by random noise that is a reflecting Brownian motion and the stochastically perturbed system of (1) is described as

$$
d x(t)=a x(t) d t+f(x(t)) d Y(t)
$$


for all $t \geq 0$, where the map $f: R \rightarrow R$ is a Borel measurable function which satisfies the local Lipschitz assumption that is to say, for every positive integer $\theta$, there is a constant $K_{\theta} \geq 0$ such that, for all $x, \tilde{x} \in R$ with $|x| \vee|\tilde{x}| \leq \theta$,

$$
|f(x)-f(\tilde{x})| \leq K_{\theta}|x-\tilde{x}| .
$$

By using Proposition 6.1 in Gonglu (1995), the reflecting Brownian motion $Y$ has a Skorohod decomposition that is

$$
Y(t)=Y(0)+\widetilde{B}(t)+A(t),
$$

where $\widetilde{B}(t)$ is a standard $\mathscr{F}_{t}$-adapted Brownian motion, $A(t)$ is a $\mathscr{F}_{t}$-adapted continuous increasing process with the initial data $A(0)=0$ a.s. Then by Theorem 1 of Bass (1990), the reflecting Brownian motion $Y$ on the half line $[0, \infty)$ is a continuous semimartingale. Based on the Skorohod decomposition (3), the system (2) turns the following system

$$
d x(t)=a x(t) d t+f(x(t)) d \widetilde{B}(t)+f(x(t)) d A(t) .
$$

The system (4) is equivalent to the stochastic integral form

$$
x(t)=x_{0}+\int_{0}^{t} a x(s) d s+\int_{0}^{t} f(x(s)) d \widetilde{B}(s)+\int_{0}^{t} f(x(s)) d A(s) .
$$

Since $f: R \rightarrow R$ satisfies the local Lipschitz assumption, by using Theorem 3.2 in Gonglu (1995), one sees that the system (5) exists a unique solution. We denote it by $x(t)$ for every $t \geq 0$. Obviously, the solution process is a continuous semimartingale. Assume further that $f(0)=0$, if the initial data $x(0)=0$, then the equation $(5)$ has the trivial solution $x(t) \equiv 0$.

The purpose of this paper is to establish the conditions under which the trivial solution of (5) can come to the stability state. To act this aim, some definitions for stochastic stability of the system shall be introduced.

First the operators $L, M, N$ associated with the equation (5) are defined by

$$
\begin{gathered}
L=\frac{\partial}{\partial t}+a x \frac{\partial}{\partial x}+\frac{1}{2} f^{2}(x) \frac{\partial^{2}}{\partial x^{2}}, \\
M=f(x) \frac{\partial}{\partial x}, \\
N=\frac{\partial}{\partial y}+f(x) \frac{\partial}{\partial x},
\end{gathered}
$$

and then the definition of exponential stability is given below.

Definition 2.1 By the Definition 3.1 in Mao (1997), the trivial solution of (5)is said to be almost surely exponentially stable if

$$
\lambda=\limsup _{t \rightarrow \infty} \frac{1}{t} \log |x(t)|<0 \quad \text { a.s. }
$$

for each $x_{0} \in R$, where $\lambda$ is called the sample Lyapunov exponent of the solution.

\section{Stochastic Stabilization}

Let $C^{2,1,1}\left(R \times R_{+} \times R_{+} ; R_{+}\right)$be the family of all functions $W: R \times R_{+} \times R_{+} \rightarrow R_{+}$, which are continuously once differentiable in $y, t \in R_{+}$and twice in $x \in R$. For every $W \in C^{2,1,1}\left(R \times R_{+} \times R_{+} ; R_{+}\right)$, according to Itô's formula for continuous semimartingales, it is reduced that

$W(x(t), A(t), t)-W\left(x_{0}, 0,0\right)=\int_{0}^{t} L W(x(s), A(s), s) d s+\int_{0}^{t} M W(x(s), A(s), s) d \widetilde{B}(s)+\int_{0}^{t} N W(x(s), A(s), s) d A(s)$.

All through this paper, the following assumption is always satisfied.

Assumption 3.1 Assume that $f$ is a function satisfied the local Lipschitz condition, $W(x, y, t) \in C^{2,1,1}\left(R \times R_{+} \times\right.$ $\left.R_{+} ; R_{+}\right)$, and that

$$
N W(x, y, t) \leq 0 .
$$

Lemma 3.2 Assume that the inequality (8) holds, then

$$
P\{x(t) \neq 0 \text { on } t \geq 0\}=1 .
$$


for all $x_{0} \neq 0$.

Proof. Assume that the lemma is not true, so there will be some $x_{0} \neq 0$ such that $P(\xi<\infty)>0$, where $\xi$ is the first time that the solution reaches the origin, i.e.

$$
\xi=\inf \{t \geq 0: x(t)=0\} .
$$

So we can find two constants $T>0$ and $\theta>1$ sufficiently large for $P(B) \neq 0$, where

$$
B=\{\xi \leq T \text { and }|x(t)| \leq \theta-1 \text { for all } 0 \leq t \leq \xi\} .
$$

For $0<|x| \leq \theta$ and $0 \leq t \leq T$, let $W(x, y, t)=|x|^{-1}$, we compute

$$
\begin{aligned}
L W(x, y, t) & =-a|x|^{-3} x^{2}+|x|^{-3} f^{2}(x) \\
& \leq\left(a+K_{\theta}^{2}\right)|x|^{-1}=M_{\theta} W(x, y, t),
\end{aligned}
$$

where $M_{\theta}=a+K_{\theta}^{2}$. The inequality is guaranteed from the assumption that $f$ satisfies the local Lipschitz condition and that $f(0)=0$.

Now for every $0<\varepsilon<\left|x_{0}\right|$, the stopping time is defined by

$$
\xi_{\varepsilon}=\inf \{t \geq 0:|x(t)| \leq \varepsilon \text { or }|x(t)| \geq \theta\} .
$$

According to Itô's formula for continuous semimartingales and the equality [7], we get that

$$
\begin{aligned}
& E\left[e^{-M_{\theta}\left(\xi_{\varepsilon} \wedge T\right)} W\left(x\left(\xi_{\varepsilon} \wedge T\right), A\left(\xi_{\varepsilon} \wedge T\right), \xi_{\varepsilon} \wedge T\right)\right] \\
& =W\left(x_{0}, 0,0\right)+E \int_{0}^{\xi_{\varepsilon} \wedge T} e^{-M_{\theta} s}\left[-M_{\theta} W(x(s), A(s), s)+L W(x(s), A(s), s)\right] d s \\
& +E \int_{0}^{\xi_{\varepsilon} \wedge T} e^{-M_{\theta} s} N W(x(s), A(s), s) d A(s) .
\end{aligned}
$$

Apply (8), (10) and $A(t) \geq 0$ one sees that

$$
E\left[e^{-M_{\theta}\left(\xi_{\varepsilon} \wedge T\right)} W\left(x\left(\xi_{\varepsilon} \wedge T\right), A\left(\xi_{\varepsilon} \wedge T\right), \xi_{\varepsilon} \wedge T\right)\right] \leq\left|x_{0}\right|^{-1} .
$$

If $\omega \in B$, then $\xi_{\varepsilon} \leq T$ and $\left|x\left(\xi_{\varepsilon}\right)\right| \leq \varepsilon$. So

$$
\begin{aligned}
E\left[e^{-M_{\theta} T} \varepsilon^{-1} I_{B}\right] & \leq E\left[e^{-M_{\theta} \xi_{\varepsilon}}\left|x\left(\xi_{\varepsilon}\right)\right|^{-1} I_{B}\right] \\
& =E\left[e^{-M_{\theta}\left(\xi_{\varepsilon} \wedge T\right)} W\left(x\left(\xi_{\varepsilon} \wedge T\right), A\left(\xi_{\varepsilon} \wedge T\right), \xi_{\varepsilon} \wedge T\right) I_{B}\right] \\
& \leq\left|x_{0}\right|^{-1} .
\end{aligned}
$$

Hence,

$$
P(B) \leq \varepsilon\left|x_{0}\right|^{-1} e^{M_{\theta} T} .
$$

When $\varepsilon \rightarrow 0, P(B)=0$, however the result goes against the definition of $B$ and the lemma holds.

Theorem 3.3 Suppose that there exists constants $p, b_{1}, b_{3} \in R_{+}, b_{2} \in R$, and a positive function $W \in C^{2,1,1}(R \times$ $\left.R_{+} \times R_{+} ; R_{+}\right)$, such that for all $x \neq 0, y>0, t>0$,

$$
\begin{gathered}
b_{1}|x|^{p} \leq W(x, y, t), \\
L W(x, y, t) \leq b_{2} W(x, y, t), \\
(M W(x, y, t))^{2} \geq b_{3} W^{2}(x, y, t),
\end{gathered}
$$

Then

$$
\lambda \leq \frac{2 b_{2}-b_{3}}{2 p} \quad \text { a.s. }
$$


for all $x_{0} \in R$. In particular, when $2 b_{2}<b_{3}$, the stochastic integral equation (5) is almost surely exponentially stable.

Proof. Obviously, (11) holds for $x_{0}=0$ since $x(t) \equiv 0$. Therefor (11) only needs to be shown for $x(0) \neq 0$. According to the Lemma 3.2, for all $t \geq 0$, we know that $x(t) \neq 0$ a.s. Due to Itô's formula for continuous semimartingales and the equality [7], for each $t \geq 0$, we therefor have that

$$
\begin{aligned}
& \log W(x(t), A(t), t)-\log W\left(x_{0}, 0,0\right) \\
& =\int_{0}^{t} \frac{L W(x(s), A(s), s)}{W(x(s), A(s), s)} d s+\int_{0}^{t} \frac{M W(x(s), A(s), s)}{W(x(s), A(s), s)} d \widetilde{B}(s) \\
& +\int_{0}^{t} \frac{N W(x(s), A(s), s)}{W(x(s), A(s), s)} d A(s)-\frac{1}{2} \int_{0}^{t}\left[\frac{M W(x(s), A(s), s)}{W(x(s), A(s), s)}\right]^{2} d s .
\end{aligned}
$$

By condition (iv) and the conditions that $W(x, y, t) \geq 0$ and $A(t) \geq 0$, we get that

$$
\begin{aligned}
& \log W(x(t), A(t), t)-\log W\left(x_{0}, 0,0\right) \\
& \leq \int_{0}^{t} \frac{L W(x(s), A(s), s)}{W(x(s), A(s), s)} d s+\int_{0}^{t} \frac{M W(x(s), A(s), s)}{W(x(s), A(s), s)} d \widetilde{B}(s)-\frac{1}{2} \int_{0}^{t}\left[\frac{M W(x(s), A(s), s)}{W(x(s), A(s), s)}\right]^{2} d s .
\end{aligned}
$$

Using condition (ii), it can be easily shown that for each $t \geq 0$,

$$
\log W(x(t), A(t), t) \leq \log W\left(x_{0}, 0,0\right)+M(t)+b_{2} t-\frac{1}{2} \int_{0}^{t}\left[\frac{M W(x(s), A(s), s)}{W(x(s), A(s), s)}\right]^{2} d s,
$$

where

$$
M(t)=\int_{0}^{t} \frac{M W(x(s), A(s), s)}{W(x(s), A(s), s)} d \widetilde{B}(s)
$$

is a continuous martingale whose initial value is $M(0)=0$. The following arguments are exactly the same as described in the page 121-122 of Mao (1997), and we get

$$
\limsup _{t \rightarrow \infty} \frac{1}{t} \log W(x(t), A(t), t) \leq \frac{1}{2}\left[2 b_{2}-(1-\varepsilon) b_{3}\right] \quad \text { a.s. }
$$

Finally, using condition $(i)$, then

$$
\lambda \leq \frac{2 b_{2}-(1-\varepsilon) b_{3}}{2 p} \quad \text { a.s. }
$$

and the required assertion (11) follows from the arbitrariness of $\varepsilon>0$. When $2 b_{2}<b_{3}$, the almost sure exponential stability of the system (5) is guaranteed by Definition 2.1. The proof is therefor complete.

Conclusion 3.4 From Theorem 3.3, it is easy to see that a one-dimensional unstable linear system can be stabilized by the reflecting Brownian motion .In contrast to the Theorem 3.3 in Mao (1997), while the random noise is Brownian motion, we get that Brownian motion can stabilize an unstable system better.

\section{An Example}

Consider a simple and unstable linear system

$$
\frac{d x(t)}{d t}=2 x(t), \quad t \geq 0
$$

and give the initial condition $x(0)=3$. Then the corresponding system perturbed by a reflecting Brownian motion is described below.

$$
d z(t)=2 z(t) d t-4 z(t) d Y(t)
$$

with the initial condition $z(0)=3$. Here the random noise $Y(t)$ is a reflecting Brownian motion with $Y(t)=|B(t)|$. And the solution of system (14) is denoted by $z(t)$ for all $t \geq 0$. Now apply Theorem 3.3 to judge whether the system (14) is stable. Let $W(z, y, t)=z^{2}$. Then

$$
L W(z, y, t)=20 z^{2},
$$




$$
\begin{gathered}
(M W(z, y, t))^{2}=64 z^{4}, \\
N W(z, y, t)=-8 z^{2}<0 .
\end{gathered}
$$

Hence, when $p=2, b_{1}=1, b_{2} \geq 20, b_{3} \leq 64$, by Theorem 3.3, the solution of (14) satisfies

$$
\lambda \leq-6<0 \quad \text { a.s. }
$$

So the system (14) turns stable, and this shows that the reflecting Brownian motion has stabilized the unstable system(13). And the following simulation (Figure 1) demonstrates the phenomenon well.

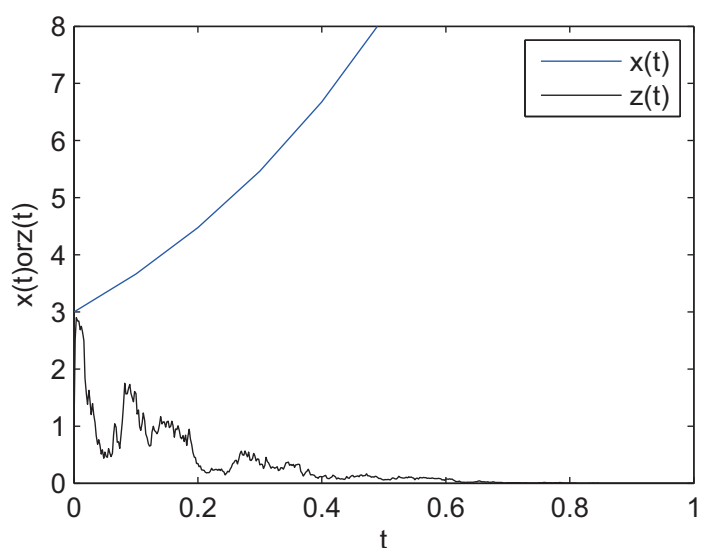

Figure 1. Simulation

\section{Acknowledgements}

The authors are thankful to Tongjun He for his help, the editor and the reviewer for the valuable comments and suggestions.

\section{References}

Arnold, L., Crauel, H., \& Wihstutz, V. (1983). Stabilization of linear system by noise. SIAM Journal on Control and Optimization, 21, 451-461. http://dx.doi.org/10.1137/0321027

Bass, R. F., \& Pei, H.(1990). The semimartingale structure of reflecting Brownian motion. American Mathematical Society, 108(4), 1007-1010. http://dx.doi.org/10.2307/2047960

David, A., \& Michailina, S. (2010). Stochastic stabilization of dynamical system using lévy noise. Stochastics and Dynamics, 10(4), 509-527. http://dx.doi.org/10.1142/S0219493710003066

Deng, H., Krstic, M., \& Williams, R. J. (2001). Stabilization of stochastic nonlinear systems driven by noise of unknown covariance. IEEE Transactions on Automatic Control, 46(8), 1237-1253. http://dx.doi.org/10.1109/9.940927

Gonglu, G. (1995). An introduce to stochastic differential equations (2nd ed.). Beijing: Beijing University Press.

Hasminskii, R. Z. (1981). Stochastic stability of differential equations. Alphen: Sijthoff and Noordhoof.

Nobuyuki, I., \& Shinzo, W. (1981). Stochastic differential equation and diffusion processes. Amsterdam: NorthHolland.

Scheutzow, M. (1993). Stabilization and destabilization by noise in the plane. Sto. Anal. Appl., 11, 97-113. http://dx.doi.org/10.1080/07362999308809304

Xuerong, M. (1994). Stochastic stabilization and destabilization. Systems and Control Letters, 23(4), 279-290. http://dx.doi.org/10.1016/0167-6911(94)90050-7

Xuerong, M. (1997). Stochastic differential equations and applications. Chichester: Horwood Publications. 